\title{
Magnetic properties of the $\mathrm{RCuIn}(\mathrm{R}=\mathrm{Ce}, \mathrm{Nd}, \mathrm{Gd}, \mathrm{Tb}, \mathrm{Dy}, \mathrm{Ho}, \mathrm{Er})$ and $\mathrm{R}_{2} \mathrm{CuIn}_{3}(\mathrm{R}=\mathrm{Ce}, \mathrm{Gd}, \mathrm{Tb}, \mathrm{Dy})$ compounds
}

\author{
A. SZYTUŁA ${ }^{1}$, Yu. TYVANCHUK ${ }^{2}$, T. JAWORSKA-GOŁABB ${ }^{1}$, A. ZARZYCKI ${ }^{1}$, Ya. KALYCHAK ${ }^{2}$, \\ Ł. GONDEK ${ }^{3}$, N. STÜSSER ${ }^{4}$ \\ ${ }^{1}$ Marian Smoluchowski Institute of Physics, Jagiellonian University, Reymonta 4, 30-059 Kraków, Poland \\ ${ }^{2}$ Department of Inorganic Chemistry, Ivan Franko National University of Lviv, \\ Kyryla i Mefodiya 6, 79005 Lviv, Ukraine \\ ${ }^{3}$ Faculty of Physics and Applied Computer Science, AGH University of Science and Technology, \\ Mickiewicza 30, 30-059 Kraków, Poland \\ ${ }^{4}$ BENSC, Hahn-Meitner Institute, Berlin-Wannsee, 14-109 Berlin, Germany \\ * Corresponding author. Tel.: +48 1266355 46; fax: +48 1263370 86; e-mail: szytula@if.uj.edu.pl
}

Received February 15, 2008; accepted February 17, 2008; available on-line March 31, 2008

X-ray diffraction and magnetic measurements of RCuIn ( $R=\mathrm{Ce}, \mathrm{Nd}, \mathrm{Gd}, \mathrm{Tb}, \mathrm{Dy}, \mathrm{Ho}, \mathrm{Er})$ and $\mathrm{R}_{2} \mathrm{CuIn}_{3}$ $(\mathbf{R}=\mathrm{Ce}, \mathrm{Gd}, \mathrm{Tb}, \mathrm{Dy})$ are reported. The RCuIn compounds crystallize in the hexagonal ZrNiAl-type structure. Two types of hexagonal structure were found in the $\mathrm{R}_{2} \mathrm{CuIn}{ }_{3}$ series of compounds: the $\mathrm{AlB}_{2}$ type for $R=C e$ and the $C a I n_{2}$ type for $R=G d, T b, D y$. DC magnetic susceptibility measurements show that all the compounds, except those with $R=C e$, order antiferromagnetically with $T_{\mathrm{N}}$ ranging from $3.1 \mathrm{~K}(\mathrm{R}=\mathrm{Er})$ to $21 \mathrm{~K}(\mathrm{R}=\mathrm{Gd})$ in the RCuIn series and from $25.6 \mathrm{~K}(\mathrm{R}=\mathrm{Dy})$ to $40 \mathrm{~K}$ for $(R=\mathrm{Tb})$ in the $R_{2} \mathrm{CuIn}$ series. Neutron diffraction measurements were carried out for TbCuIn. At 1.5 K the Tb magnetic moments lie in the basal plane and form a noncollinear magnetic structure described by the propagation vector $k=(1 / 2,1 / 2$, $0.2217(9))$. The frustration effect characteristic of a triangular lattice is observed.

Rare earth alloys and compounds / Intermetallics / Magnetically ordered materials / Magnetic measurements / Neutron diffraction

\section{Introduction}

The $\mathrm{R}-\mathrm{Cu}$-In systems ( $\mathrm{R}$ is a rare earth element) have been thoroughly investigated. Isothermal sections of the phase diagrams and crystallographic data for series of ternary compounds are collected in the review article [1]. According to the information therein, from 4 up to 10 intermetallic compounds are formed in each system and the $\mathrm{Ce}-\mathrm{Cu}$-In system contains the highest number of compounds.

Intermetallic phases containing 33.3 at. $\% \mathrm{R}$ are the subject of the present work. Compounds of the RCuIn stoichiometry are known for all rare earths except Eu and $\mathrm{Yb}$. They crystallize in the hexagonal $\mathrm{ZrNiAl}$ structure type (space group $P-62 m$ ), where the $\mathrm{R}, \mathrm{Cu}$ and In atoms occupy the positions of $\mathrm{Zr}$ (the $3 g$ site: $x_{\mathrm{R}}, 0,1 / 2$ ), Ni (the $1 b: 0,0,1 / 2$ and $2 c: 1 / 3,2 / 3,0$ sites) and Al (the $3 f: x_{\text {In }}, 0,0$ site), respectively. For Er, Tm and $\mathrm{Lu}$ the compounds form limited homogeneity ranges as a result of In substitution for $\mathrm{Ni}$ [1,2]. Compounds of the $\mathrm{R}_{2} \mathrm{CuIn}_{3}$ (or $\mathrm{RCu}_{0.5} \mathrm{In}_{1.5}$ ) stoichiometry are known for all rare earths except $\mathrm{Yb}$ and $\mathrm{Lu}$. All these compounds have solubility regions along the isoconcentration line of the rare earth element [1]. The crystal structure of $\mathrm{CeCu}_{0.5} \mathrm{In}_{1.5}$ has been solved as an example of a representative of the $\mathrm{AlB}_{2}$-type structure. The $\mathrm{R}$ atoms occupy the positions of the $\mathrm{Al}$ atoms, while the positions of the $\mathrm{B}$ atoms are occupied by a statistical mixture of $\mathrm{Cu}$ and $\mathrm{In}$ atoms $\left(\mathrm{Cu}_{0.5} \mathrm{In}_{1.5}\right)$ [3].

Magnetic properties in the RCuIn series have been investigated only for $\mathrm{R}=\mathrm{Ce}, \mathrm{Gd}$ and $\mathrm{Tb}$. It was reported that CeCuIn is a paramagnet down to $4.2 \mathrm{~K}$ [4], GdCuIn orders antiferromagnetically below $T_{\mathrm{N}}=20 \mathrm{~K}$ [5], while the magnetic susceptibility of TbCuIn shows two maxima (at 16 and $84 \mathrm{~K}$ ) in the temperature dependence [6].

Neutron diffraction data for TbCuIn collected in the temperature range $1.5 \mathrm{~K}$ and $50 \mathrm{~K}$ confirm a hexagonal crystal structure at $50 \mathrm{~K}$. At $1.5 \mathrm{~K}$ the $\mathrm{Tb}$ magnetic moments form a noncollinear magnetic structure described by the propagation vector $\mathbf{k}=(1 / 2,1 / 2,0.2288(3))[6]$.

Magnetic measurements carried out for $\mathrm{R}_{2} \mathrm{CuIn}_{3}$ showed that the compounds with $\mathrm{R}=\mathrm{Nd}, \mathrm{Tb}, \mathrm{Dy}$, Ho and $\mathrm{Er}$ order antiferromagnetically with the Néel temperature $T_{\mathrm{N}}$ ranging from $8 \mathrm{~K}(\mathrm{R}=\mathrm{Ho})$ to $30 \mathrm{~K}$ 
$(\mathrm{R}=\mathrm{Tb})$ [7]. According to neutron diffraction measurements the $\mathrm{Tb}$ magnetic moments, which equal $6.8 \mu_{\mathrm{B}}$ at $\mathrm{T}=3.8 \mathrm{~K}$, form a collinear antiferromagnetic structure described by the propagation vector $\mathbf{k}=(0,1 / 2,0)$. The $\mathrm{Tb}$ magnetic moments are parallel to the $c$-axis $[8,9]$.

This paper presents magnetic data collected for two series of rare earth compounds: $\mathrm{RCuIn}(\mathrm{R}=\mathrm{Ce}, \mathrm{Nd}$, Gd-Er) and $\mathrm{R}_{2} \mathrm{CuIn}_{3}(\mathrm{R}=\mathrm{Ce}, \mathrm{Gd}, \mathrm{Tb}, \mathrm{Dy})$. For TbCuIn neutron diffraction was carried out to determine the magnetic structure.

\section{Experimental}

Polycrystalline samples were prepared by arc melting of high-purity raw metals (rare earths with a purity not worse than 99.8 wt.\% main component, electrolytic copper with the purity 99.92 wt. $\% \mathrm{Cu}$ and indium with the purity $99.99 \mathrm{wt} \%$ In) in titanium-gettered argon atmosphere $(\mathrm{p}=50 \mathrm{kPa})$. The chemical composition of the alloys was checked by measuring the weight losses, which did not exceed $1 \mathrm{wt} . \%$. The total mass of each ingot was about $1.5 \mathrm{~g}$. All the ingots were annealed at $870 \mathrm{~K}$ for $700 \mathrm{~h}$ in evacuated silica tubes and then quenched in cold water.

$\mathrm{X}$-ray powder diffraction patterns were collected at room temperature using $\mathrm{CuK}_{\alpha}$ radiation (Philips X'PERT diffractometer; Institute of Physics of the Jagiellonian University). Neutron diffraction measurements of TbCuIn were carried out in the temperature range $1.5 \div 50 \mathrm{~K}$ with the incident neutron wavelength $2.44 \AA$ (E6 diffractometer at the Berlin Neutron Scattering Center). The diffraction data were analyzed using the Rietveld-type program Fullprof [10]. DC magnetic measurements were carried out in the temperature range $2 \div 300 \mathrm{~K}$ in magnetic fields up to 50 kOe (MPMS SQUID magnetometer; Institute of Physics of the Jagiellonian University).

\section{Results}

The X-ray analysis confirms that all the obtained RCuIn alloys are single phase with ZrNiAl-type structures. A typical X-ray diffraction pattern is shown in Fig. 1. Crystallographic data calculated by full-profile analysis [10] of the X-ray diffraction patterns are collected in Table 1 and agree well with published data [1].

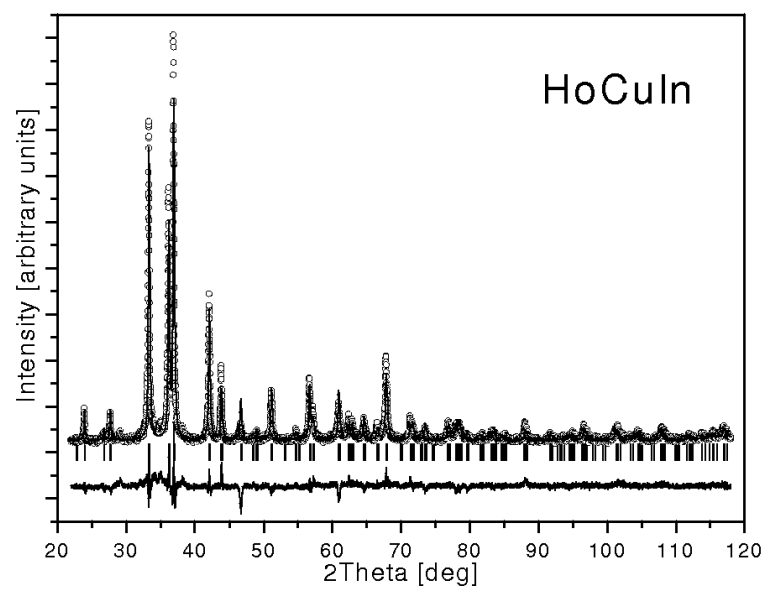

Fig. 1 X-ray powder diffraction pattern of HoCuIn.

The crystal structure parameters of TbCuIn determined from the neutron diffraction data collected at $50 \mathrm{~K}$ (paramagnetic region) are as follows: $a=7.404(3) \AA$, $c=3.946(2) \AA, x_{\mathrm{R}}=0.5923(1)$ and $x_{\mathrm{In}}=0.2397(1)$. For the $\mathrm{R}_{2} \mathrm{CuIn}_{3}$ compounds the solubility regions are known but only alloys with $\mathrm{R}=\mathrm{Ce}, \mathrm{Gd}, \mathrm{Tb}$ and $\mathrm{Dy}$ were successfully prepared for the investigations. The $\mathrm{X}$-ray analysis of the samples confirms that they are single phase and the diffraction patterns can be indexed using a hexagonal $\mathrm{AlB}_{2}$ - or $\mathrm{CaIn}_{2}$-type structure model. Values of the refined cell parameters are listed in Table 2 and are in good agreement with published ones [1].

Results of the magnetic measurements are presented in Figs. 2 and 3. In both series of compounds investigated here the Ce compounds are paramagnets down to $2 \mathrm{~K}$. All the other compounds are antiferromagnets with the Néel temperature between $21 \mathrm{~K}(\mathrm{R}=\mathrm{Gd})$ and $3.1 \mathrm{~K}(\mathrm{R}=\mathrm{Er})$ for $\mathrm{RCuIn}$, and $40 \mathrm{~K}$ $(\mathrm{R}=\mathrm{Gd})$ and $25.6 \mathrm{~K}(\mathrm{R}=\mathrm{Dy})$ for $\mathrm{R}_{2} \mathrm{CuIn}_{3}$. The anomaly at $89 \mathrm{~K}$ observed for GdCuIn is connected with a $\mathrm{Gd}_{2} \mathrm{Cu}_{2}$ In impurity, which is a ferromagnet [11]. Except for GdCuIn, the reciprocal magnetic susceptibility obeys the Curie-Weiss law above the Néel temperature, the values of the

Table 1 Crystallographic data for RCuIn compounds (X-ray diffraction at room temperature).

\begin{tabular}{l|l|l|l|l|r|r}
\hline \multirow{2}{*}{ Compound } & \multicolumn{2}{|c|}{ Cell parameters $(\AA)$} & \multicolumn{2}{c|}{ Atom coordinates } & \multicolumn{2}{c}{ Reliability factors $(\%)$} \\
\cline { 2 - 7 } & \multicolumn{1}{|c|}{$a$} & \multicolumn{1}{c|}{$c$} & \multicolumn{1}{c}{$x_{\mathrm{R}}$} & $x_{\mathrm{In}}$ & $\mathrm{R}_{\text {Bragg }}$ & $\mathrm{R}_{\mathrm{F}}$ \\
\hline CeCuIn & $7.4915(18)$ & $4.2452(15)$ & $0.5832(12)$ & $0.2486(15)$ & 14.7 & 11.0 \\
NdCuIn & $7.4715(9)$ & $4.1668(6)$ & $0.5874(9)$ & $0.2462(12)$ & 9.7 & 8.0 \\
GdCuIn & $7.4701(15)$ & $3.9925(9)$ & $0.5897(21)$ & $0.2528(21)$ & 13.0 & 9.8 \\
TbCuIn & $7.4586(9)$ & $3.9633(6)$ & $0.5881(12)$ & $0.2524(12)$ & 15.0 & 12.8 \\
DyCuIn & $7.4486(15)$ & $3.9297(12)$ & $0.5914(15)$ & $0.2464(18)$ & 13.5 & 11.3 \\
HoCuIn & $7.4332(9)$ & $3.8911(6)$ & $0.5911(9)$ & $0.2506(9)$ & 12.0 & 8.2 \\
ErCuIn & $7.4247(6)$ & $3.8637(6)$ & $0.5912(9)$ & $0.2524(12)$ & 11.9 & 11.9 \\
\hline
\end{tabular}


paramagnetic Curie temperature are negative and the effective magnetic moments are close to the free $\mathrm{R}^{3+}$ ion values. At $T=2 \mathrm{~K}$ and the magnetic field $\mathrm{H}=50$ kOe the values of the magnetic moments are smaller than the free $\mathrm{R}^{3+}$ ion values. The results of the magnetic measurements are listed in Table 3.

Table 2 Cell parameters of the $\mathrm{R}_{2} \mathrm{CuIn}_{3}$ compounds (X-ray diffraction at room temperature).

\begin{tabular}{c|l|l|l}
\hline \multirow{2}{*}{ Compound } & \multirow{2}{*}{$\begin{array}{c}\text { Structure } \\
\text { type }\end{array}$} & \multicolumn{2}{|c}{ Cell parameters $(\AA)$} \\
\cline { 3 - 4 } & \multicolumn{1}{|c}{$a$} & \multicolumn{1}{c}{$c$} \\
\hline $\mathrm{Ce}_{2} \mathrm{CuIn}$ & \multicolumn{1}{c}{$c$} \\
$\mathrm{Gd}_{2} \mathrm{CuIn}$ & & $4.819(2)$ & $3.874(1)$ \\
$\mathrm{Tb}_{2} \mathrm{CuIn}$ & $\mathrm{CaIn}_{2}$ & $4.7409(9)$ & $7.3857(9)$ \\
$\mathrm{Dy}_{2} \mathrm{CuIn}$ & $\mathrm{CaIn}_{2}$ & $4.7358(6)$ & $7.3209(6)$ \\
\hline
\end{tabular}
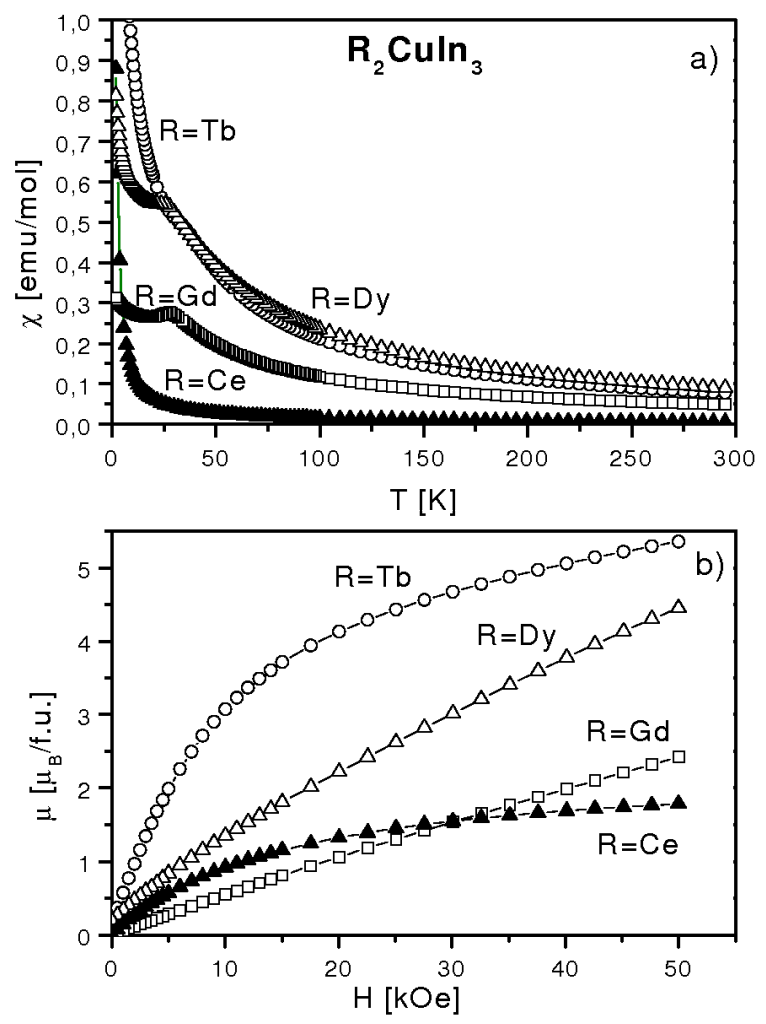

Fig. 2 (a) Temperature dependence of the magnetic susceptibility and (b) magnetization curve at $2 \mathrm{~K}$ for $\mathrm{RCuIn}(\mathrm{R}=\mathrm{Ce}, \mathrm{Nd}, \mathrm{Gd}, \mathrm{Tb}$, Dy, Ho, Er) compounds.

Analysis of the neutron diffraction pattern of TbCuIn collected at $1.5 \mathrm{~K}$ confirms a noncollinear magnetic structure described by the propagation vector $\mathbf{k}=(1 / 2,1 / 2,0.2217(9))$ and magnetic moments in the basal plane. The temperature dependence of the magnetic peak intensities gives a Néel temperature equal $14.5 \mathrm{~K}$. In the $\mathrm{ZrNiAl}$-type structure the $\mathrm{Tb}$ magnetic moments are located at the following positions: $\mu_{1}$ at $\left(x_{\mathrm{R}}, 0,1 / 2\right), \mu_{2}$ at $\left(\bar{x}_{R}, \bar{x}_{R}, 1 / 2\right)$ and $\mu_{3}$ at $\left(0, x_{\mathrm{R}}, 1 / 2\right)$. The best fit of the magnetic part of the neutron diffraction pattern was obtained for the magnetic structure described below:

- the magnetic moment $\mu_{1}$ equal $4.9(2) \mu_{\mathrm{B}}$ and slightly tilted from the $a$-axis direction,

- $\mu_{2}$ equal 4.9(2) $\mu_{\mathrm{B}}$ and perpendicular to the $a$-axis,

- $\mu_{3}$ equal 5.5(2) $\mu_{\mathrm{B}}$ and parallel to the $b$-axis.

The basal plane projection of the magnetic structure of TbCuIn is shown in Fig. 4. The magnetic structure determined here is consistent with the one presented in [6].
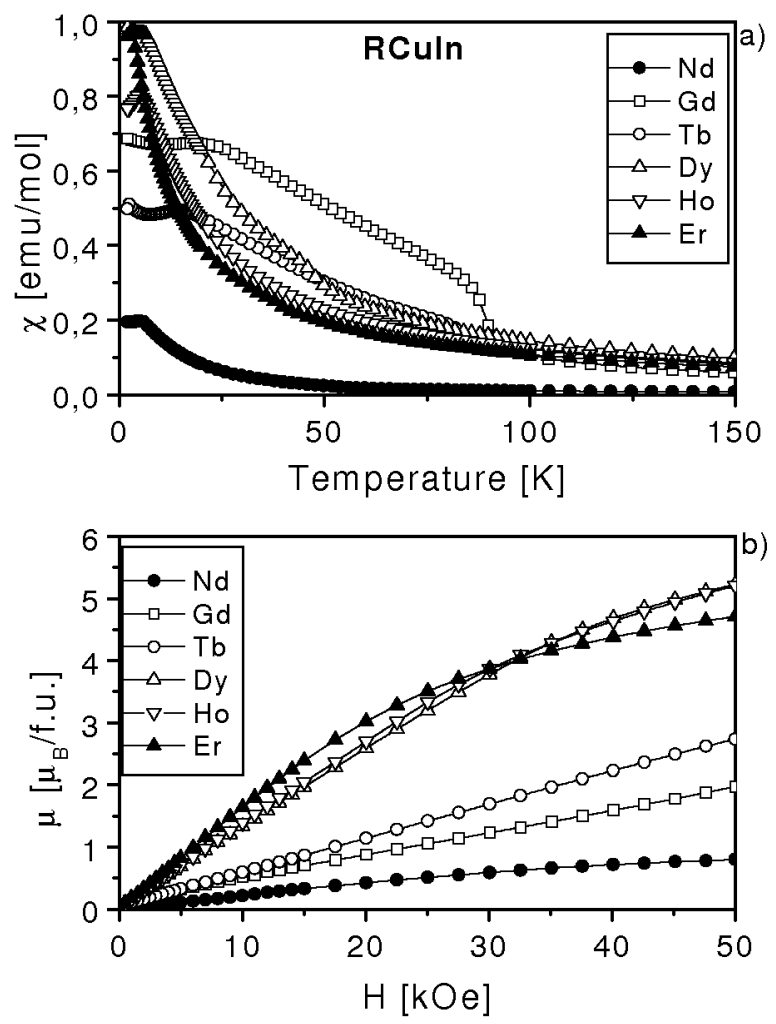

Fig. 3 (a) Temperature dependence of the magnetic susceptibility and (b) magnetization curve at $2 \mathrm{~K}$ for $\mathrm{R}_{2} \mathrm{CuIn}_{3}(\mathrm{R}=\mathrm{Ce}, \mathrm{Gd}, \mathrm{Tb}, \mathrm{Dy})$ compounds.

\section{Discussion and conclusions}

The results presented in this work confirm that the RCuIn compounds crystallize in hexagonal crystal structures of the $\mathrm{ZrNiAl}$ type and the $\mathrm{R}_{2} \mathrm{CuIn}_{3}$ compounds have hexagonal structures of the $\mathrm{CaIn}_{2}$ $(\mathrm{R}=\mathrm{Gd}, \mathrm{Tb}$ and $\mathrm{Dy})$ or $\mathrm{AlB}_{2}$ type $(\mathrm{R}=\mathrm{Ce})$. The investigated compounds (except $\mathrm{R}=\mathrm{Ce}$ ) are antiferromagnets at low temperatures. The data for the RCuIn series are, in majority, the first results published concerning the magnetic properties of these compounds. The data for $\mathrm{R}_{2} \mathrm{CuIn}_{3}$ are in good agreement with the data reported in [7]. Contrary to the $\mathrm{R}_{2} \mathrm{Cu}_{2}$ In compounds [11], in the RCuIn and $\mathrm{R}_{2} \mathrm{CuIn}_{3}$ compounds the magnetic moments order antiferromagnetically. 
Table 3 Magnetic properties of the RCuIn and $\mathrm{R}_{2} \mathrm{CuIn}_{3}$ series of compounds.

\begin{tabular}{l|c|c|c|c|c|c|c|c|c|c}
\hline \multirow{2}{*}{$\mathrm{R}$} & \multicolumn{4}{|c|}{$\mathrm{RCuIn}$} & \multicolumn{3}{c|}{$\mathrm{R}_{2} \mathrm{CuIn}_{3}$} & \multicolumn{2}{c}{$\mathrm{R}^{3+}$} \\
\cline { 2 - 11 } & $T_{\mathrm{N}}(\mathrm{K})$ & $\Theta_{\mathrm{p}}(\mathrm{K})$ & $\mu_{\mathrm{eff}}\left(\mu_{\mathrm{B}}\right)$ & $\mu\left(\mu_{\mathrm{B}}\right)$ & $T_{\mathrm{N}}(\mathrm{K})$ & $\Theta_{\mathrm{p}}(\mathrm{K})$ & $\mu_{\mathrm{eff}}\left(\mu_{\mathrm{B}}\right)$ & $\mu\left(\mu_{\mathrm{B}}\right)$ & $\mu_{\mathrm{eff}}\left(\mu_{\mathrm{B}}\right)$ & $\mu\left(\mu_{\mathrm{B}}\right)$ \\
\hline $\mathrm{Ce}$ & $\mathrm{P}^{\mathrm{a}}$ & -15.0 & 2.40 & 0.7 & $\mathrm{P}^{\mathrm{a}}$ & -8.6 & 2.52 & 1.8 & 2.54 & 2.14 \\
$\mathrm{Nd}$ & 4.9 & -7.2 & 2.96 & 0.8 & & & & & 3.58 & 3.2 \\
$\mathrm{Gd}$ & 21.0 & 6.8 & 8.27 & 2.0 & 27.4 & -31.9 & 7.93 & 2.4 & 7.94 & 7.0 \\
$\mathrm{~Tb}$ & 14.5 & -5.4 & 9.44 & 2.7 & 40.0 & -12.3 & 9.77 & 5.4 & 9.72 & 9.0 \\
$\mathrm{Dy}$ & 6.2 & -6.6 & 10.52 & 5.2 & 25.6 & -20.5 & 10.65 & 4.4 & 10.65 & 10.0 \\
$\mathrm{Ho}$ & 5.0 & -10.0 & 10.51 & 5.2 & & & & & 10.61 & 10.0 \\
$\mathrm{Er}$ & 3.1 & -12.3 & 9.93 & 4.7 & & & & & 9.58 & 9.0 \\
\hline
\end{tabular}

a paramagnetic down to $2 \mathrm{~K}$

In the compounds investigated in this work the magnetic moments are localized on the rare earth atoms. The interatomic distances between these atoms are long, which suggests that the interaction between the magnetic moments is of the RKKY type. In this model the Néel temperatures $T_{\mathrm{N}}$ should follow the de Gennes factor $(g-1)^{2} \mathrm{~J}(\mathrm{~J}+1)$. According to the de Gennes scaling, $T_{\mathrm{N}}$ should reach its maximum for $\mathrm{Gd}$ compounds. The dependence of the $T_{\mathrm{N}}$ value on the de Gennes factor for both investigated systems is plotted in Fig. 5 (data for $\mathrm{R}_{2} \mathrm{CuIn}_{3}(\mathrm{R}=\mathrm{Er}$, Ho) from [7] are included). It is seen that the de Gennes scaling (marked by a dashed line in Fig. 5) is fulfilled for the RCuIn compounds, while for the $\mathrm{R}_{2} \mathrm{CuIn}_{3}$ compounds the maximum of the Néel temperature is shifted from $\mathrm{Gd}$ to $\mathrm{Tb}$, which may result from crystalline electric field (CEF) effects [12]. The smaller values of the $\mathrm{Tb}$ magnetic moments in the ordered state in comparison to the paramagnetic one could also be explained as the result of the CEF. The influence of the CEF is manifested in the different directions of the $\mathrm{Tb}$ magnetic moments: in the basal plane for TbCuIn, but along the $c$-axis for $\mathrm{Tb}_{2} \mathrm{CuIn}_{3}$ and for the isostructural $\mathrm{Tb}_{2} \mathrm{AgIn}_{3}$ [13]. The values of the Néel temperatures are higher for the $\mathrm{R}_{2} \mathrm{CuIn}_{3}$ compounds, which suggests that the CEF effect strengthens the RKKY interaction.

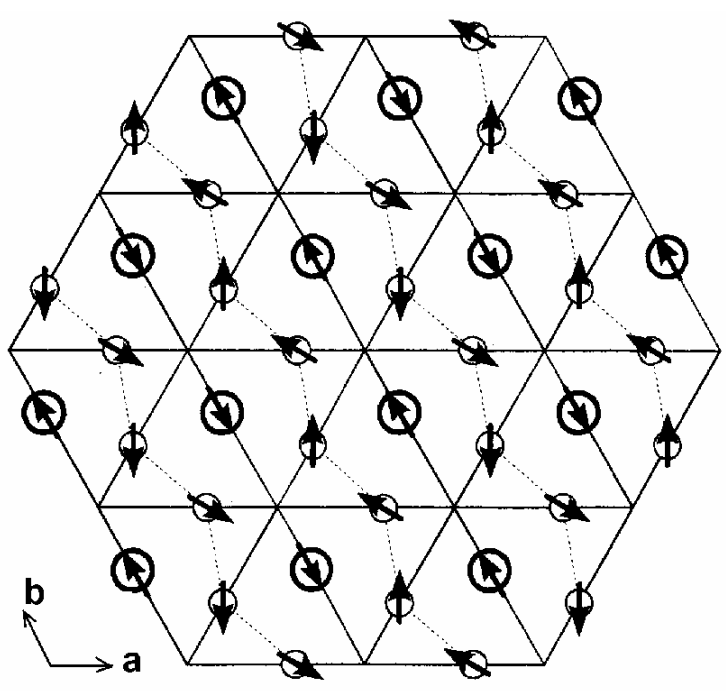

Fig. 4 Basal plane projection of the magnetic structure of TbCuIn.

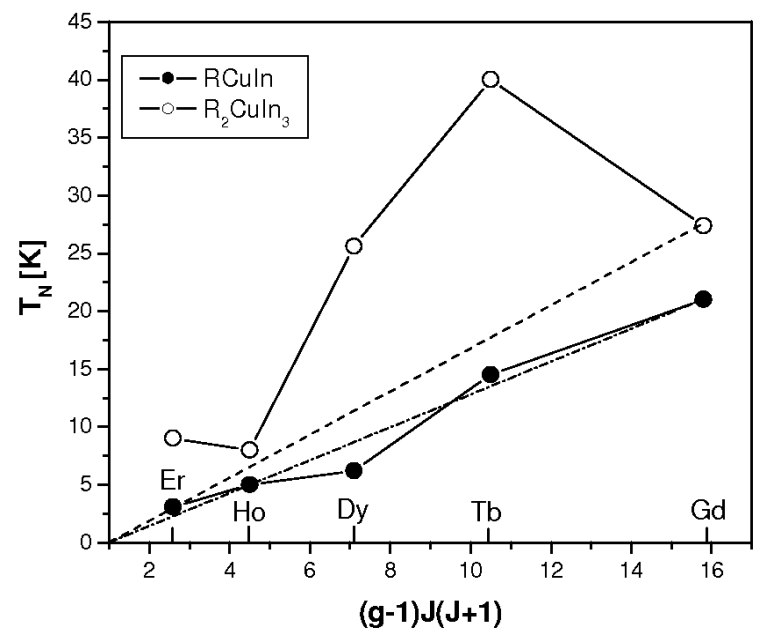

Fig. 5 Néel temperature vs. the de Gennes factor $(\mathrm{g}-1)^{2} \mathrm{~J}(\mathrm{~J}+1)$ for RCuIn and $\mathrm{R}_{2} \mathrm{CuIn}_{3}$ compounds.

In both $\mathrm{TbCuIn}$ and $\mathrm{Tb}_{2} \mathrm{CuIn}_{3}$ the frustration effect characteristic of a triangular lattice is observed. In TbCuIn the Tb moments build a noncollinear structure typical for a triangular lattice. In this structure neighbouring rare earth magnetic moments form an angle of $120^{\circ}$ with each other (see Fig. 4). In $\mathrm{Tb}_{2} \mathrm{CuIn}_{3}$ a simple collinear magnetic structure with an orthorhombic unit cell $(a, \sqrt{3} a, c)$ is developed. This magnetic ordering is in good agreement with the Isinglike model for a two-dimensional hexagonal lattice with a strong anisotropy (that forces the magnetic moment to align parallel to the $c$-axis), taking into account the exchange integrals between the first $\left(\mathrm{J}_{1}\right)$ and the second $\left(\mathrm{J}_{2}\right)$ nearest neighbours.

Based on these predictions Doukouré et al. [14] obtained the magnetic phase diagram. The magnetic structure of $\mathrm{Tb}_{2} \mathrm{CuIn}_{3}$ corresponds to the $\mathrm{J}_{1}<0$ and $\mathrm{J}_{2}<0$ or $\mathrm{J}_{1}>0$ and $\mathrm{J}_{2} \geq-\mathrm{J}_{1}$ set of exchange integrals.

\section{Acknowledgements}

This work was supported by the European Commission under the Sixth Framework Programme through the Key Action: Strengthening the European Research Area, Research Infrastructures Contract: 
H113-CT-2003-505925 (NMI3). Two of the authors (Ł.G. \& A.Sz.) would like to express their gratitude to the management of the BENSC for financial support and kind hospitality. Yu.T. would like to acknowledge the financial support from the Józef Mianowski Found and kind hospitality of the Institute of Physics of the Jagiellonian University.

\section{References}

[1] Ya.M. Kalychak, Izv. Akad. Nauk SSSR, Metally 4 (1998) 110 (in Russian).

[2] P. Villars, L.D. Calvert, Pearson's Handbook of Crystallographic Data for Intermetallic Phases, ASM International, Materials Park, 1991.

[3] V.M. Baranyak, O.V. Dmytrakh, Ya.M. Kalychak, P.Yu. Zavalij, Izv. Akad. Nauk SSSR, Neorg. Mater. 24 (1988) 873 (in Russian).

[4] S.K. Malik, D.T. Adroja, B.D. Padalia, R. Vijavaghavan, Physica B 163 (1990) 89.
[5] J.W.C. de Vries, R.C. Thiel, K.H.J. Buschow, J. Less-Common Met. 111 (1985) 313.

[6] G. Ehlers, Thesis, Techn. Univ. Berlin, 1996.

[7] I.M. Siouris, I.P. Semitelou, J.K. Yakinthos, J. Alloys Compd. 297 (2000) 26.

[8] I.M. Siouris, I.P. Semitelou, J.K. Yakinthos, W. Schäfer, R.R. Arons, J. Alloys Compd. 314 (2001) 1.

[9] I.M. Siouris, I.P. Semitelou, J.K. Yakinthos, R.R. Arons, W. Schäfer, J. Magn. Magn. Mater. 226230 (2001) 1128.

[10] J. Rodriguez-Carvajal, Program FullProf, Laboratoire Leon Brillouin (CEA-CNRS), 2000.

[11] I.R. Fisher, Z. Islam, P.C. Canfield, J. Magn. Magn. Mater. 202 (1999) 1.

[12] N.T. Hutchings, Solid State Phys. 16 (1964) 227.

[13] I.P. Semitelou, I.M. Siouris, J.K. Yakinthos, W. Schäfer, D. Schmitt, J. Alloys Compd. 283 (1999) 12.

[14] M. Doukouré, D. Gignoux, F. Sayatat, Solid State Commun. 58 (1986) 713.

Proceeding of the IX International Conference on Crystal Chemistry of Intermetallic Compounds, Lviv, September 20-24, 2005. 